\title{
Prevalence of redetectable positive SARS-CoV-2 nucleic acid in recovered COVID-19 patients: a systematic review and meta-analysis
}

\author{
Xiaoshuang Shi ${ }^{1}$, Wencui Xiu ${ }^{2}$, Weijuan Gang ${ }^{2}$, Jiwei Yang ${ }^{2}$, Yixuan Feng ${ }^{2}$, Lanping Liu ${ }^{2}$, \\ Jinxin $\mathrm{Ma}^{2}$, Xia $\mathrm{Li}^{2}$, Lanjun $\mathrm{Shi}^{2}$, Zhiyun Zhang ${ }^{2}$, Qingquan $\mathrm{Yu}^{2}$, and Xianghong Jing ${ }^{2}$ \\ ${ }^{1}$ China Academy of Chinese Medical Sciences Institute of Acupuncture and Moxibustion \\ ${ }^{2}$ Affiliation not available
}

April 30, 2021

\begin{abstract}
Background: The prevalence of positive SARS-CoV-2 nucleic acid in recovered COVID-19 patients has attracted attention. We aimed to investigate the repositive rate of SARS-CoV-2 and the clinical features of discharged COVID-19 patients. Methods: In this systematic review and meta-analysis, we searched PubMed, Embase, Web of Science, China National Knowledge Internet, Wanfang Data, China BioMedical Literature, VIP, and Google Scholar. Fixed or random-effect models were used to determine effect estimates. Results: Eleven studies were included. The pooled positive rate of viral RNA in discharged patients was $11 \%$ (95\% CI $7-15$; I2=90.4\%). The median days from discharge to repositivity were 7 to 8 days. Coughing was the most common clinical symptom, occurring in $16 \%(95 \%$ CI 11-20; I2=0\%) of patients at readmission. Chest CT and laboratory indicators of positive retest $(\mathrm{PR})$ patients showed significant recovery trends. The prevalence of comorbidities between the PR patients and the negative retest patients were not significant (OR 0.86 [95\% CI 0.38-1.95]; $\mathrm{p}=0.002 ; \mathrm{I} 2=76.5 \%$ ). No close contacts were positive for SARS-CoV-2 RNA. Conclusion: PR patients were uncommon. The repositive result was likely due to the incomplete clearance of virus from a previous disease course. PR patients were less likely to be contagious. However, close monitoring and quarantine after discharge from the hospital are necessary. Registration: The protocol has been registered on PROSPERO, registration ID: CRD42021239650 Keywords: COVID-19, SARS-CoV-2, discharged patients, positive retest rate
\end{abstract}

\section{Hosted file}

manuscript-upload.pdf available at https://authorea.com/users/410965/articles/520174prevalence-of-redetectable-positive-sars-cov-2-nucleic-acid-in-recovered-covid-19patients-a-systematic-review-and-meta-analysis 
14673

Records identified through darabase seaching

PubMed: 4433

Embase: 4410

Web of Science: 942

Google Scholar: 4570

CNKI: 132

Wanfang Data: 60

VIP: 71

CBM: 55

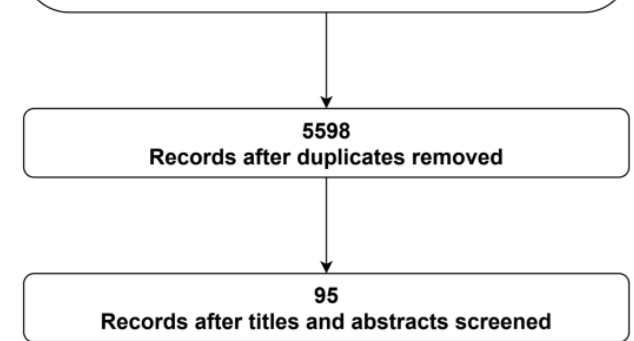

Records after titles and abstracts screened

Full text articles excluded

2 Research letter

1 Review

80 Not relevant studies

1 Duplication 

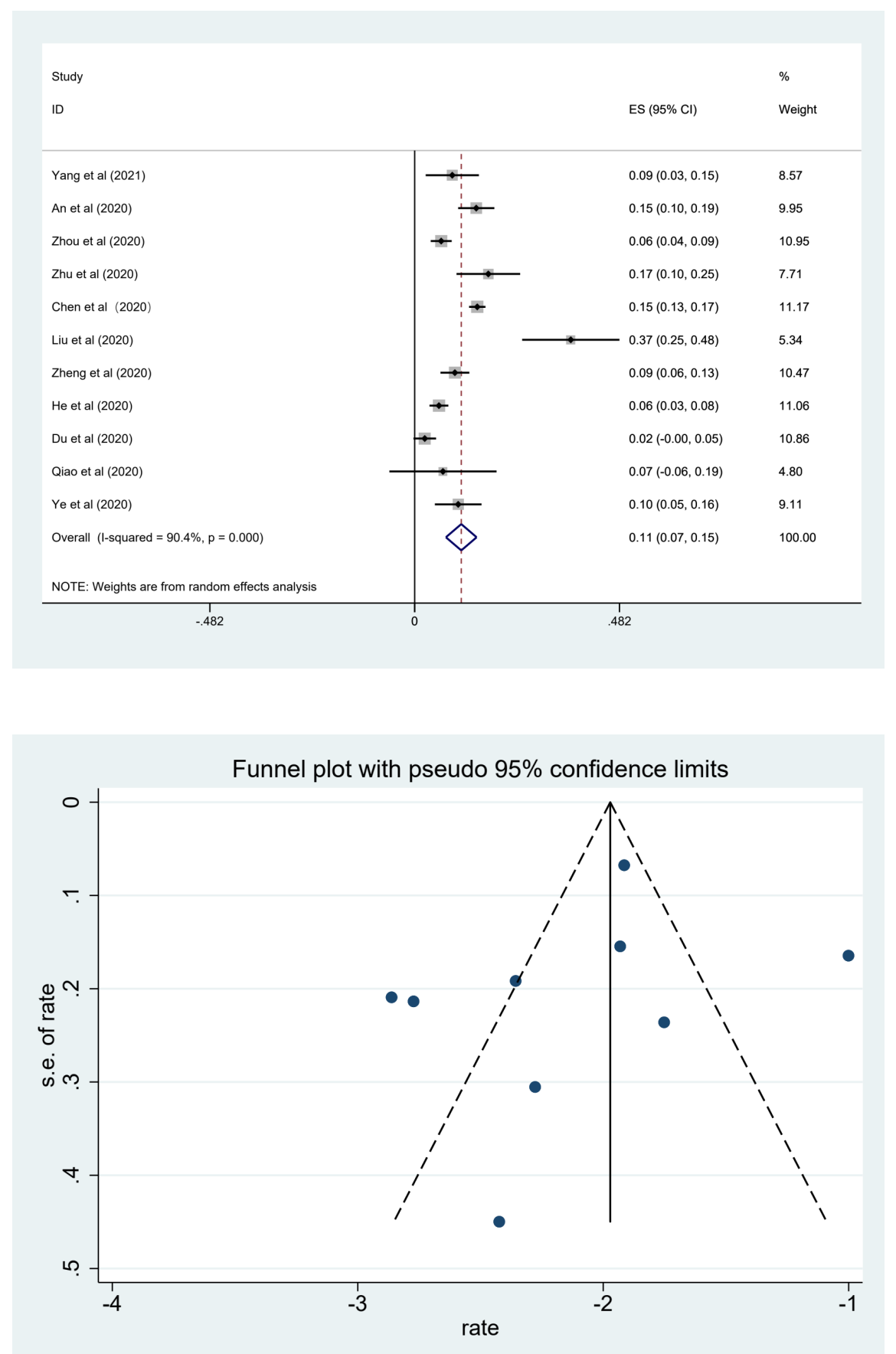